\title{
Aerobic Training Modulates the Increase in Plasma Concentrations of Cytokines in response to a Session of Exercise
}

\author{
Tatiana Ramos Fonseca, ${ }^{1,2}$ Thiago Teixeira Mendes, ${ }^{1,3}$ Guilherme Passos Ramos, ${ }^{1}$ \\ Christian Emmanuel Torres Cabido, ${ }^{1,3}$ Rodrigo Figueiredo Morandi, ${ }^{1}$ \\ Fernanda Oliveira Ferraz, ${ }^{2}$ Aline Silva Miranda, ${ }^{2,4}$ Vanessa Amaral Mendonça, ${ }^{2,5}$ \\ Antônio Lúcio Teixeira, ${ }^{4}$ Emerson Silami-Garcia, ${ }^{1,3}$ Albená Nunes-Silva $\mathbb{D}^{6},{ }^{6,7}$ \\ and Mauro Martins Teixeira ${ }^{2}$ \\ ${ }^{1}$ Laboratório de Fisiologia do Exercício, Departamento de Educação Física (LAFISE/EEFFTO/UFMG), Belo Horizonte, Brazil \\ ${ }^{2}$ Laboratório de Imunofarmacologia, Departamento de Bioquímica e Imunologia (ICB/UFMG), Belo Horizonte, Brazil \\ ${ }^{3}$ Departamento de Educação Física (UFMA), São Luís, Brazil \\ ${ }^{4}$ Laboratório Interdisciplinar de Investigação Médica, Faculdade de Medicina (UFMG), Belo Horizonte, Brazil \\ ${ }^{5}$ Laboratório de Inflamação e Metabolismo (LIM/UFVJM), Teófilo Otoni, Brazil \\ ${ }^{6}$ Laboratório de Resolução da Resposta Inflamatória, Departamento de Morfologia (ICB/UFMG), Belo Horizonte, Brazil \\ ${ }^{7}$ Laboratório de Inflamação e Imunologia do Exercício (LABIIEx), Escola de Educação Física da Universidade \\ Federal de Ouro Preto (UFOP), Ouro Preto, Brazil \\ Correspondence should be addressed to Albená Nunes-Silva; albenanunes@hotmail.com
}

Received 18 May 2020; Revised 19 November 2020; Accepted 31 December 2020; Published 16 January 2021

Academic Editor: Amir Radfar

Copyright (c) 2021 Tatiana Ramos Fonseca et al. This is an open access article distributed under the Creative Commons Attribution License, which permits unrestricted use, distribution, and reproduction in any medium, provided the original work is properly cited.

\begin{abstract}
Acute physical exercise can modulate immune function. For example, acute exercise is known to increase the circulating concentration of cytokines. Exercise is also known to modulate immune function chronically. It is not known whether exercise training can result in training of the immune system. Here, we investigated the effects of six weeks of aerobic training on cytokine responses induced by acute exercise until fatigue. Twelve healthy men performed a fatiguing exercise at the anaerobic threshold (AT) intensity. After the training period, the participants performed another bout of acute exercise at the same duration and intensity of the pretraining situation. The analysis was made at the beginning, end, and at 10, 30, and 60 minutes during the recovery period. Training at AT induced a gain of $11.2 \%$ of exercise capacity. Before training, a single bout of acute exercise induced a significant increase in plasma levels of cytokines, including IL-6, TNF- $\alpha$, sTNFR1, IL-10, CXCL10, BDNF, leptin, resistin, and adiponectin. After six weeks of aerobic training, levels of IL-6, sTNFR1, BDNF, and leptin increased to a lesser extent after an acute bout exercise at the same absolute intensity as the pretraining period. Responses to the same relative exercise intensity were similar to those observed before exercise. These results show that aerobic training is associated with training of acute immune responses to acute exercise until fatigue.
\end{abstract}

\section{Introduction}

Regular physical exercise is thought to have important beneficial effects on human health. Indeed, over the past three decades, a significant body of evidence has demonstrated that regular physical exercise induces considerable changes in the health of the population [1-3]. In addition, the use of exercise has become a staple in the prevention and treatment options for delaying the development of health issues, such as overweight and obesity [3]. Studies have also shown that there is a strong association between the increase in physical inactivity and the emergence of modern chronic diseases in 20th century industrialized societies [1], and lack of exercise is considered a major risk factor for chronic 
diseases [2]. Many aspects of human health can be affected by exercise, including cardiovascular risk reduction [4], insulin sensitivity [5] and blood glucose homeostasis [6], weight loss and maintenance [7], and systemic inflammation [8-12]. In addition, physical exercise improves memory acquisition [13], regulates mood and anxiety, works as a strategy for reducing depression, enhances well-being [14], increases life longevity [15], improves learning [16], and improves cardiorespiratory fitness and biomarkers of cardiometabolic health [17].

Many studies have now clearly shown that many types of acute physical exercise induce remarkable alterations in the behavior of the immune system [18-25]. Acute physical exercise modifies the number and function of the circulating leukocyte pool $[26,27]$. In addition, it has been shown that plasma level of many cytokines increases during and after a single acute exercise session [28-32]. The increase in the level of gene expression and local and circulating protein content is associated with the type and intensity of exercise and the health status of participants [33]. Different exercise protocols and load induce distinct responses in the concentration of cytokines in plasma [34-37].

Therefore, there is a large body of convincing evidence showing an interaction between physical exercise and the immune system (as seen by the systemic elevation of cytokines) during and after an acute physical exercise session [38-41]. This cytokine response is believed to be necessary for the formation of new vessels and the remodeling of skeletal muscle [42] and adipose tissue [43].

Chronic physical training is known to be associated with a gain of exercise capacity. However, it is not known whether physical training will impact on acute cytokine responses to an acute bout of exercise. The hypothesis of this study is that the acute increase in plasma levels of cytokines in response to an acute bout intense exercise is modulated by physical training. Fundamentally, this study aims to contribute to the concept that it is possible to train the innate immune system [44] and that exercise is an important physiological manner to do so.

Thus, the purpose of this study was to investigate the effects of physical exercise training on the modulation of cytokine responses to an acute bout of exercise. Indeed, it was our objective to ascertain whether exercise training would induce not only physical training, but also the "training" of the immune system, as assessed by measuring levels of cytokines in response to acute exercise stimulation.

To this end, we evaluated whether six weeks of aerobic training program would modify the cytokine (IL-6, TNF- $\alpha$, sTNFR1, IL-10, CXCL10, BDNF, leptin, resistin, and adiponectin) responses induced by an acute bout of exercise performed until fatigue at anaerobic threshold intensity.

\section{Materials and Methods}

2.1. Participants and Study Design. Twelve young $(22.5 \pm 0.7$ years) untrained males volunteered to participate, after recruitment through posters and social media at our university. Mean $\left( \pm\right.$ SEM) body mass, height, and $\mathrm{VO}_{2 \text { PEAK were }}$ $71.9 \pm 1.6 \mathrm{~kg}, 1.76 \pm 0.02 \mathrm{~m}$, and $45.5 \pm 1.2 \mathrm{~mL} \cdot \mathrm{kg}^{-1} \cdot \mathrm{min}^{-1}$, respectively. All participants were also asked to keep a food log of the $24 \mathrm{~h}$ preceding each acute exercise session. All procedures in this investigation were performed in accordance with the Declaration of Helsinki, Ethics Committee of Federal University of Minas Gerais approved all procedures (EC\# 261/09), and all participants provided written informed consent after reading a detailed description of the protocol.

At pretraining, all participants were tested to determine $\mathrm{VO}_{2 \text { PEAK }}$ and the anaerobic threshold (AT) (pretraining/ intensity 1, I1) and performed acute exercise until fatigue at I1. There was a minimum of $48 \mathrm{~h}$ of rest between all exercise sessions and $72 \mathrm{~h}$ before the test until fatigue. Following sixweek aerobic training program, all participants were subjected to retesting to define the posttraining $\mathrm{VO}_{2 \mathrm{PEAK}}$ and AT (posttraining/intensity 2, I2). After a minimum of $72 \mathrm{~h}$ for last training session, all participants performed two sessions of acute exercise (in different days and intensities): the first session had similar duration and absolute intensity $(w)$ of initial test (pretraining) at I1 (to develop a similar total work when compared to the pretraining situation), and the second session was performed at I2 until fatigue (experimental design figure) (to develop a similar relative intensity at AT, due to training adaptations). All tests were always performed at the same time of the day $( \pm 1 \mathrm{~h})$ to avoid circadian effects and in a room with controlled temperature and humidity $\left(21-24^{\circ} \mathrm{C}, 50-70 \% \mathrm{RH}\right)$.

Tests for physical characterization and tests until fatigue (time to fatigue) at anaerobic threshold (AT) before (AT-I1) and after (AT-I2) a six-week aerobic training. $\mathrm{VO}_{2 \mathrm{PEAK}}$ : maximal oxygen peak; AT: anaerobic threshold, AT-I1: intensity of AT before training; AT-I2: intensity of AT posttraining. Note. Posttraining participants performed two tests until fatigue at different intensities: at AT-I2 and at ATI1 (with similar duration on pretraining).

The participants performed 6 weeks of aerobic training, three times per week, at the I1 (about 75\% VO $\mathrm{VO}_{2 \mathrm{PEAK}}$ ). The duration of the first training session was $24 \mathrm{~min}$, and it increased to $39 \mathrm{~min}$ over the 6-week period (increase of 3 min per week) (Table 1). The training protocol was adapted from the study by Philp et al. [45], and the six-week period seems to be sufficient to induce significant adaptations $[45,46]$ in untrained participants. Each training session took place under the supervision of a researcher. All tests and aerobic training were performed on a cycle ergometer (Monark Ergomedic E-824E) previously calibrated, according to the manufacturer specifications, before each test and training.

Body mass and height were measured with a digital scale and a stadiometer (Filizola ${ }^{\circledR}$ ). The body composition was estimated by the seven-skinfold method according to Jackson and Pollock [47]. The same researcher always performed the skinfold evaluation with a skinfold caliper (Lange ${ }^{\circledR}$ ).

The participants were asked to drink $500 \mathrm{~mL}$ of water two hours prior to the beginning of all situations (experimental situations and training sessions) [48]. In all experimental situations, all participants were instructed to not drink any alcohol or beverages containing caffeine or perform any acute physical activity for at least 24 hours prior to 
TABLE 1: The progression of six-week aerobic training program.

\begin{tabular}{llr}
\hline Week & Frequency $\left(\right.$ days week $\left.^{-1}\right)$ & Time $(\mathrm{min})$ \\
\hline 1 & 3 & 24 \\
2 & 3 & 27 \\
3 & 3 & 30 \\
4 & 3 & 33 \\
5 & 3 & 36 \\
6 & 3 & 39 \\
\hline
\end{tabular}

the experiment's situation, to maintain the same diet in the evenings and mornings of the experimental situation, and each participant was asked about the experimental recommendations for food, alcohol, and caffeine consumption before starting experimental procedures. They were allowed to ingest water ad libitum in all experimental situations and training sessions, and before experimental situations, all participants were euhydrated (urine specific gravity $\leq 1020$ ).

2.2. Assessment of $V O_{2 P E A K}$. Progressive exercise to fatigue, according to Balke and Ware [49], was performed to determine the $\mathrm{VO}_{2 \text { PEAK }}$ before and after the aerobic training. The power started at $50 \mathrm{~W}$ and the workload was increased by $25 \mathrm{~W} \cdot 2 \mathrm{~min}^{-1}$. At the end of each stage, the rate of perceived exertion (RPE) according to Borg [50] was evaluated. Before each test, the participants rested sitting on a chair $(5 \mathrm{~min})$ to set the equipment for $\mathrm{O}_{2}$ uptake analysis (K4b2; Cosmed ${ }^{\circledR}$, Italy) and heart rate (HR) (Polar Team System, Finland). Ventilation variables were measured, breath-by-breath, and the gas analyzer was calibrated before each test. The highest $\mathrm{VO}_{2}$ of the exercise was considered the $\mathrm{VO}_{2 \text { PEAK}}$.

At least two of the following criteria had to be met to determine the $\mathrm{VO}_{2 \mathrm{PEAK}}$ : (1) no increase in $\mathrm{VO}_{2}$ or $\mathrm{HR}$ despite increased exercise intensity, (2) RPE greater than 17 on the 6-20 scale, and (3) respiratory exchange ratio greater than 1.10 [51].

\subsection{Assessment of AT and Responses at Acute Exercise until} Fatigue. The AT was determined as described in Mendes et al. [52] and was considered the exercise intensity of maximal lactate steady state (MLSS). The MLSS is considered for some authors the gold standard protocol to determine AT [53-55] and an intensity that could be maintained for about $45-60 \mathrm{~min}$ [56] that is probably able to induce immune response. All participants performed two to five $30 \mathrm{~min}$ exercise sessions at constant intensities to determine the AT with a minimum of $48 \mathrm{~h}$ between each test. During the AT tests, blood samples $(30 \mu \mathrm{L})$ were collected from the ear lobe for blood lactate concentration analysis prior to the beginning of the exercise and at $5 \mathrm{~min}$ intervals until the end of the test. The highest power output at which the blood lactate concentration increased to less than $1 \mathrm{mM}$ during the last 20 min of exercise was defined as the MLSS/ AT $[53,54]$. The AT was determined with a precision of $15 \mathrm{~W}$. Blood was stored at $-20^{\circ} \mathrm{C}$ in tubes containing $60 \mu \mathrm{L}$ $\mathrm{NaF}(1 \%)$ and later analyzed in duplicate for lactate concentration using an electroenzymatic analyzer (YSI 1500 STAT, Yellow Springs, Ohio, USA).
The AT was evaluated before and after the training program (intensity I and II for pre- and posttraining, I1 and I2) and corresponded to $\sim 75 \% \mathrm{VO}_{2 \text { PEAK}}$. The $\mathrm{AT}$ was assessed to relativize the exercise intensity and to create an exercise until fatigue length of 30-60 minutes and similar times of exercise before and after training.

For the acute exercises (pretraining at I1, posttraining at I1, and posttraining at I2), fatigue was considered as an individual's inability to maintain pedal frequency of $60 \mathrm{rpm}$, RPE of 20 , or the test interruption requested by the subject. Venous blood samples were collected before $(0 \mathrm{~min})$ and at the end of the exercise (End) and at 10, 30, and 60 minutes during the recovery period to determine plasma levels of cytokines, chemokines, and lactate. Participants were not allowed to know any physiological variables, nor their exercise time, throughout all tests.

2.4. Correction for Plasma Volume Shifts and ELISA. Peripheral venipuncture was performed with a catheter (Angiocath ${ }^{\circledR}$, BD-Becton Dickinson, 22G, USA). The blood was then centrifuged, and the plasma was stored in Eppendorf tubes in a freezer at $-20^{\circ} \mathrm{C}$. Changes in plasma volume were calculated from measurements of hemoglobin and hematocrit according to the method described by Dill and Costill [57], and plasma levels of cytokines and chemokines measurements were corrected accordingly.

Plasma cytokines and chemokines were measured by ELISA according to the procedures supplied by the manufacturer (DuoSet, R \& D Systems, Minneapolis, MN, USA). The results were presented in picograms per $\mathrm{ml}(\mathrm{pg} / \mathrm{mL})$. IL6 , TNF- $\alpha$, sTNFR1, IL-10, CXCL10/IP-10, BDNF, leptin, resistin, and adiponectin were measured in this study. Blood lactate was analyzed using an electroenzymatic analyzer (YSI 1500 STAT, Yellow Springs, Ohio, USA).

2.5. Statistical Analysis. Normality was assessed using the Ryan-Joiner test, homoscedasticity was assessed using Levene's test, and sphericity was examined using Mauchly's test prior to all analyses. Cytokines and chemokines data did not achieve normal distribution and were analyzed by nonparametric tests. The variable IL-6 had normal distribution after logarithmic transformation.

To analyze the effects of the training program in the $\mathrm{VO}_{2 \text { PEAK }}$, body mass, and percentage of body fat (before and after training) paired $t$-test was used. Additionally, Cohen's $d$ effect-size (ES) estimates were calculated to assess the magnitude of difference between the experimental conditions. ES was calculated through mean differences [58] and 
was considered trivial $(\mathrm{ES}<0.2)$, small $(\mathrm{ES}: 0.2-0.6)$, medium (ES: 0.6-1.2), or large ( $E S \geq 1.2$ ) [59]. Additionally, Cohen's effect size (ES) and confidence intervals of ES (CI) of $95 \%$ were calculated to assess the magnitude of difference between the experimental conditions..

To compare exercise intensities and physiological responses for exercise sessions at pretraining at I1 and posttraining at I1 and I2, one-way ANOVA with repeated measures was used. To analyze IL-6 $(\log )$ responses, two-way ANOVA with repeated measures was used. A Student-Newman-Keuls post hoc analysis was used when necessary. To analyze other cytokines and chemokines (TNF- $\alpha$, sTNFR1, IL-10, CXCL10, BDNF, leptin, resistin, and adiponectin), a nonparametric Friedman test was applied. To test the association between the variables, a Pearson product-moment correlation coefficient (parametric-log IL-6) was used.

The results of parametric analysis are presented as mean \pm standard error of mean and nonparametric analysis of cytokines, and chemokines are presented as median and individual response. The significance level used was $p<0.05$. Statistical analysis was performed in Sigma Plot v. 13 and SPSS v. 22.

\section{Results}

3.1. Aerobic Training. Twelve healthy participants performed six weeks of aerobic training on a cycle ergometer at their anaerobic threshold (AT) before the training session (referred to as I1). We compared anthropometric parameters before and after the period of aerobic training, and no differences were detected in body mass $(71.9 \pm 1.7$ vs $71.4 \pm 1.6 \mathrm{~kg}, \mathrm{ES}=-0.30 ; \mathrm{IC}=-1.55-0.94)$ and percentage of body fat $(14.2 \pm 1.5$ vs $13.2 \pm 1.3 \%$, ES $=-0.71$; $\mathrm{IC}=-1.99-0.57)$. All the participants completed six weeks (18 sessions) of aerobic training, and this was associated with an increase of $11.2 \pm 6.6 \%$ in $\mathrm{VO}_{2 \mathrm{PEAK}}(44.9 \pm 4.8$ vs. $49.8 \pm 4.5 ; p<0.05 ; \mathrm{ES}=1.06 ; \mathrm{IC}=-0.27-2.38)$ (Figure $1(\mathrm{a})$ ) and in maximal power (POTMAX) (218.7 \pm 30.8 vs. $252.4 \pm 27.9, \quad p<0.05 ; \quad \mathrm{ES}=1.15 ; \quad \mathrm{IC}=-0.19-2.49)$ (Figure 1(b)), suggesting that aerobic training was effective in generating cardiovascular and pulmonary adaptations.

All participants were subjected to acute exercise (cycle ergometer), as a stressor response, before training (pretraining) and 6 weeks after exercise training at AT intensities. Physiological responses to exercise at I1 and posttraining I1 and 12 sessions are shown in Table 2. During the posttraining I1 session, participants performed at lower percentage of maximal oxygen uptake $\left(\% \mathrm{VO}_{2 \mathrm{PEAK}}\right)$ and maximal power $(\%$ POTMAX) when compared to the posttraining I2 session. In addition, there were lower values of RPE and HR (Table 2) in the posttraining session I1, demonstrating that the physiological stress appeared to be lower during the posttraining session I1. The latter findings are consistent with the gain in performance observed in Figure 1. In addition, the data suggest that the physiological stress was similar in pretraining at I1 and posttraining at I2 sessions.

In order to investigate the effects of aerobic training on blood circulating cytokines, we evaluated levels of cytokines previously known to elevate after acute exercise [60]. At rest, there were no detectable differences in blood concentration of the evaluated cytokines, IL- 6 , TNF- $\alpha$, sTNFR1, IL-10, CXCL10, BDNF, leptin, resistin, and adiponectin, at pretraining, posttraining I1, and posttraining I2. Levels of cytokines increased significantly at the end of an acute bout of exercise or immediately thereafter (Figures 2-10). As shown in greater detail below, it is important to notice the different behavior of certain cytokines when comparing the response to an acute bout of exercise before and after a period of training.

3.1.1. IL-6. IL-6 peaked at the very end and remained high until $60 \mathrm{~min}$ after the end of acute exercise (Figure 2). Levels of IL- 6 at the end of exercise had a positive correlation with duration of the exercise $(r=0.88 ; p=0.01$; ES = 3.71) (data not shown). After the training period, all individuals were subjected to acute exercise to the same intensity as before training (posttraining-I1). As seen in Figure 2, the concentration of IL- 6 increased after exercise, but the median increase was significantly lower and dropped considerably faster when compared to the pretraining situation. When the individuals were exposed to the new level of I2 (posttraining I2), the profile and median concentration of the IL-6 response were similar to those observed in the pretraining situation at I1 (Figure 2). Levels of IL-6 at the end of exercise at post-I2 had a positive correlation with duration of the exercise $(r=0.88 ; p=0.01 ; \mathrm{ES}=3.71)$ (Figure 3 )

3.1.2. TNF- $\alpha$. There was an increase of TNF- $\alpha$ levels only at 60 min after the end of exercise, and the intensity of increase was similar in all three groups (Figure 3); i.e., the level of TNF- $\alpha$ increased only 60 minutes after exercise, and median elevation was similar in the situation before and after training. There were no changes in IL- $1 \beta$ induced by acute exercise under our experimental conditions (data not shown).

3.1.3. sTNFR1. Plasma concentrations of sTNFR1 elevated immediately after exercise and remained high at 10, 30, and 60 minutes after the finish of acute exercise (Figure 3). After six weeks of aerobic training, level of sTNFR1 also increased in the posttraining I1 situation but returned to the basal level faster than the pretraining situation (Figure 4). When the participants were exposed to the new level of I2 (posttraining I2), the profile and concentration of the sTNFRq response were similar to those observed in the pretraining situation at I1 (Figure 2).

3.1.4. IL-10. IL-10 was only elevated 10 minutes after acute exercise, and the size of the response was similar in all three situations, i.e., before and after training and at the two levels of intensity (Figure 5).

3.1.5. CXCL10/IP-10. We evaluated the effects of acute exercise on the levels of the three chemokines: CXCL8/IL-8, CCL2/MCP-1, and CXCL10/IP-10. Acute exercise did not significantly affect the circulating levels of CXCL8/IL-8 and 


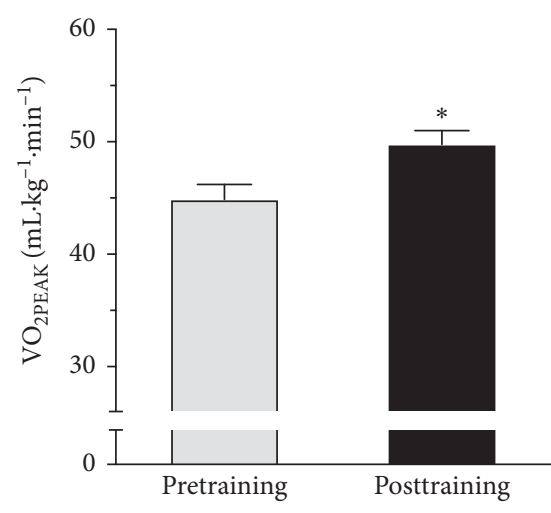

(a)

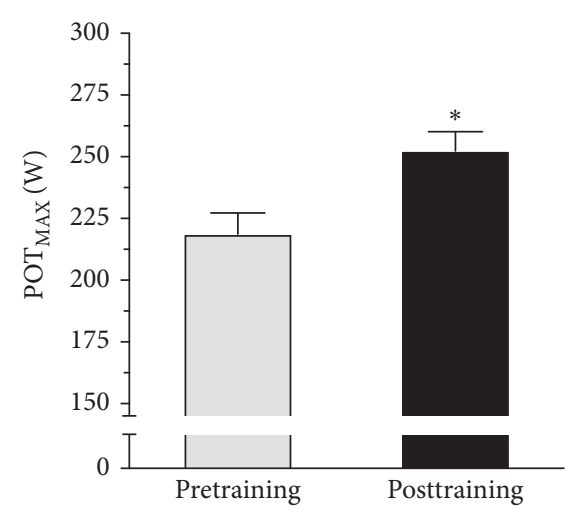

(b)

FIGURE 1: (a) Maximal oxygen uptake $\left(\mathrm{VO}_{2 \mathrm{PEAK}}\right)$ and (b) maximal power output (POTMAX) before (pre) and after (post) aerobic training. ${ }^{*} p<0.01$.

TABLE 2: Data regardingAbsolute exercise intensity (POT), blood lactate concentration (LAC), heart rate (HR), rate of perceived exertion (RPE), percentage of maximal oxygen uptake (\% $\left.\mathrm{VO}_{2 \mathrm{PEAK}}\right)$, and maximal power (\% POTMAX).

\begin{tabular}{lccc}
\hline Variables & Pretraining at I1 & Posttraining at I1 & Posttraining at I2 \\
\hline Absolute potency POT (W) & $150 \pm 8$ & $150 \pm 8$ & $171 \pm 8^{\text {\&\# }}$ \\
Relative potency \% POTMAX & $69 \pm 3$ & $59 \pm 2^{\&}$ & $68 \pm 2^{\#}$ \\
Relative intensity \% VO 2PEAK & $73.2+3.6$ & $66.1 \pm 3.0^{\&}$ & $71.9 \pm 1.6$ \\
Duration of exercise (min) & $71.9 \pm 7.5$ & $71.9 \pm 7.5$ & $65.4 \pm 7.0$ \\
Lactate (mM) & $6.4 \pm 0.5$ & $4.1 \pm 0.3^{\&}$ & $6.4 \pm 0.4$ \\
RPE & 15 & $11^{\&}$ & $14^{\#}$ \\
HR (bpm) & $158 \pm 4$ & $147 \pm 3^{\&}$ & $156 \pm 3^{\#}$ \\
\hline
\end{tabular}

${ }^{\&} p<0.05$, significantly different from pretraining at I1; ${ }^{\#} p<0.05$, significantly different from posttraining at I1. $n=10\left(\% \mathrm{VO}_{2 \mathrm{PEAK}}\right.$ at posttraining I2).

CCL2/MCP-1 under our experimental conditions (data not shown). Therefore, these chemokines were not evaluated after aerobic training. However, acute exercise induced elevation of the levels of CXCL10/IP-10 in plasma in an interesting bimodal manner. The level of IL-10 first peaked at 10 minutes after exercise; then, it returned to baseline, and another peak of greater intensity was observed at 60 minutes after exercise (Figure 6). Overall, the response was similar in the three groups; i.e., exercise-induced elevations of CXCL10 were not altered by training.

3.1.6. BDNF. As seen in Figure 7, resting ( $0 \mathrm{~min})$ levels of $\mathrm{BDNF}$ were similar in all sessions, suggesting that baseline levels of this cytokine were not altered by aerobic training. In response to acute exercise, plasma levels of BDNF increased significantly and peaked at the end of exercise and at 10 minutes of the recovery period. Overall, levels of BDNF at the peak were twice as high as those at baseline. Thereafter, levels of BDNF fell and reached baseline levels again at 60 minutes after recovery. In the posttraining I1 session, in which individuals were subjected to equal absolute potency but a decreased stressor stimulus, levels of BDNF still increased at the end of exercise and at 10 minutes of recovery. However, the increase was significantly lower than that observed in the pretraining session (Figure 7).

In the posttraining $\mathrm{I} 2$ session, in which individuals were subjected to a similar relative potency and to a similar stressor stimulus, levels of BDNF curve peaked at the end of exercise, and this was similar to that of the pretraining session. However, levels of BDNF dropped rapidly and were significantly different from those found in the pretraining session at all times of recovery.

3.1.7. Leptin. Acute physical exercise until fatigue was accompanied by an increase in plasma concentrations of leptin that peaked at the end of physical exercise and then again at 60 minutes during postexercise recovery (Figure 8). In the posttraining period, there was no significant increase in the concentration of leptin when these individuals were exposed to the same absolute intensity of the pretraining period (posttraining I1) (Figure 8). On the other hand, exposing participants to the same relative intensity of the pretraining until fatigue (posttraining I2) resulted in plasma concentrations of leptin, which were similar to those concentrations measured during acute exercise stimulation at the pretraining session.

3.1.8. Resistin. Acute physical exercise was associated with increase of plasma resistin concentrations at the end of exercise and then at 90 minutes of postexercise recovery period (Figure 9). The same kinetics and concentration of resistin were observed after training when individuals were exposed to the same absolute or relative intensity of acute exercise of the pretraining period. 


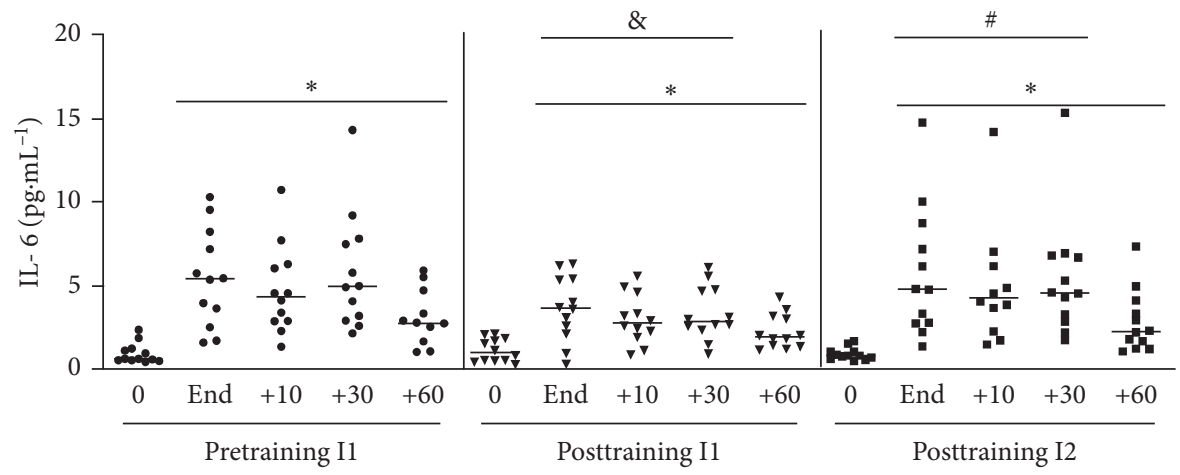

(a)

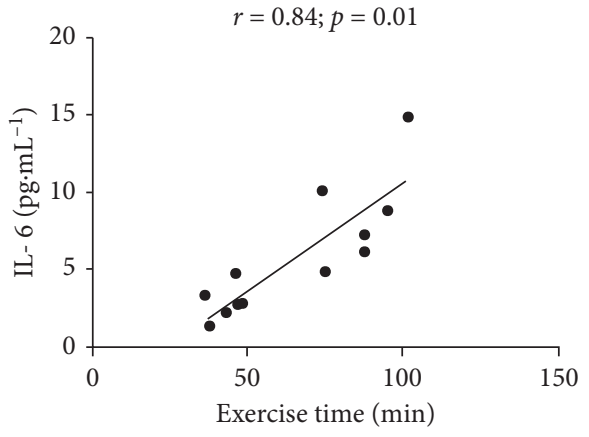

(b)

Figure 2: (a) IL-6 plasmatic concentration at pretraining and posttraining, before, after, 10, 30, and 60 min after acute exercise. Pretraining (physical exercise performed until fatigue at anaerobic threshold (I1) before 6 weeks of training); posttraining 1 (aerobic acute exercise performed at the same absolute intensity (I1) and duration of pretraining) and posttraining 2 (after aerobic acute exercise performed until fatigue at anaerobic threshold of posttraining (I2)). ${ }^{*} p<0.05$ from resting period (0) for the same situation; ${ }^{*} p<0.05$ from pretraining for the same time point; ${ }^{\#} p<0.05$ from posttraining I1 for the same time point. $n=12$. (b) Pearson product-moment correlation between IL- 6 and exercise time to fatigue. $n=12$.

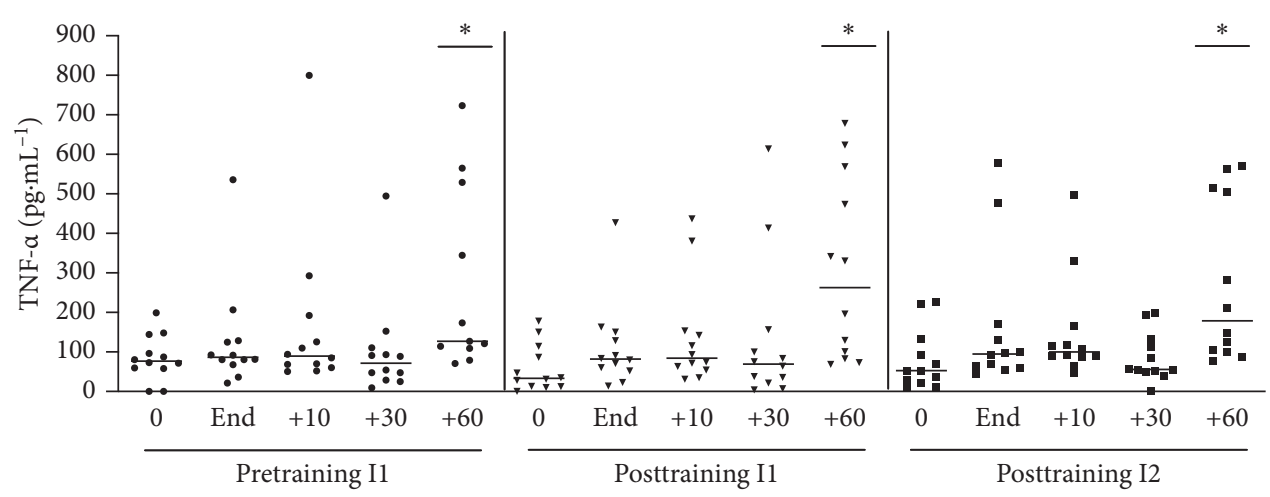

FIGURE 3: TNF- $\alpha$ plasmatic concentration at pretraining and posttraining, before, after, 10, 30, and 60 min after acute exercise. Pretraining (physical exercise performed until fatigue at anaerobic threshold (I1) before 6 weeks of training); posttraining 1 (aerobic acute exercise performed at the same absolute intensity (I1) and duration of pretraining) and posttraining 2 (after aerobic acute exercise performed until fatigue at anaerobic threshold of posttraining (I2)). ${ }^{*} p<0.05$ from resting period (0) for the same situation. $n=12$.

3.1.9. Adiponectin. Adiponectin concentration increased after acute physical exercise, only 10 minutes after exercise, in participants with no training (Figure 10). A similar profile was observed after 6 weeks of aerobic training with the same absolute or relative intensity of pretraining acute exercise stimulation.

\section{Discussion}

The major findings of the current study can be summarized as follows: (i) A six-week period of aerobic training at AT (around $75 \% \mathrm{VO}_{2 \mathrm{PEAK}}$ ) was able to induce performance improvement. (ii) Acute exercise at AT until fatigue induced 


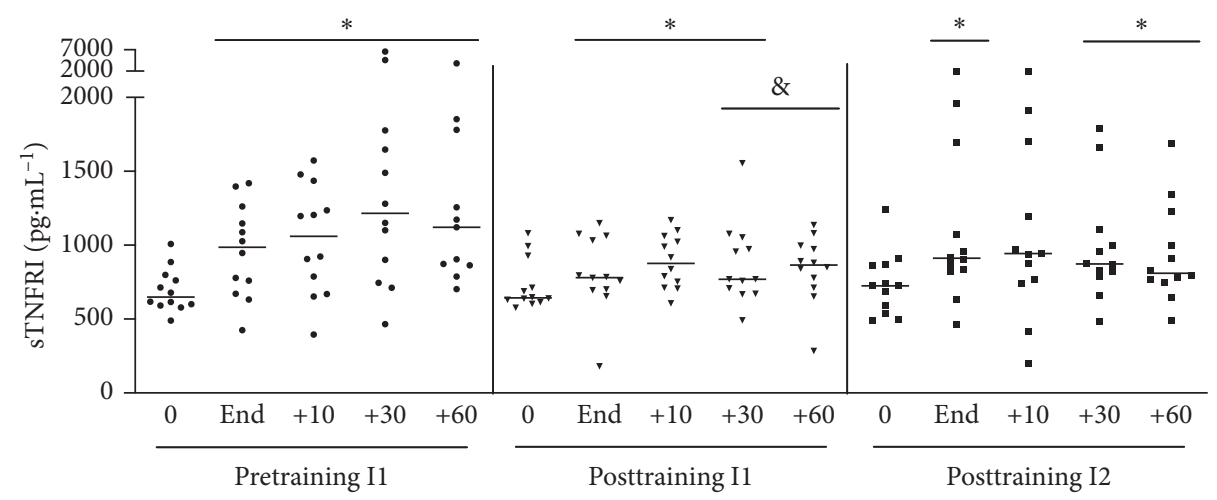

FIGURE 4: sTNFR1 plasmatic concentration at pretraining and posttraining, before, after, 10, 30, and 60 min after acute exercise. Pretraining (physical exercise performed until fatigue at anaerobic threshold (I1) before 6 weeks of training); posttraining 1 (aerobic acute exercise performed at the same absolute intensity (I1) and duration of pretraining) and posttraining 2 (after aerobic acute exercise performed until fatigue at anaerobic threshold of posttraining (I2)). ${ }^{*} p<0.05$ from resting period (0) for the same situation; ${ }^{*} p<0.05$ from pretraining for the same time point. $n=12$.

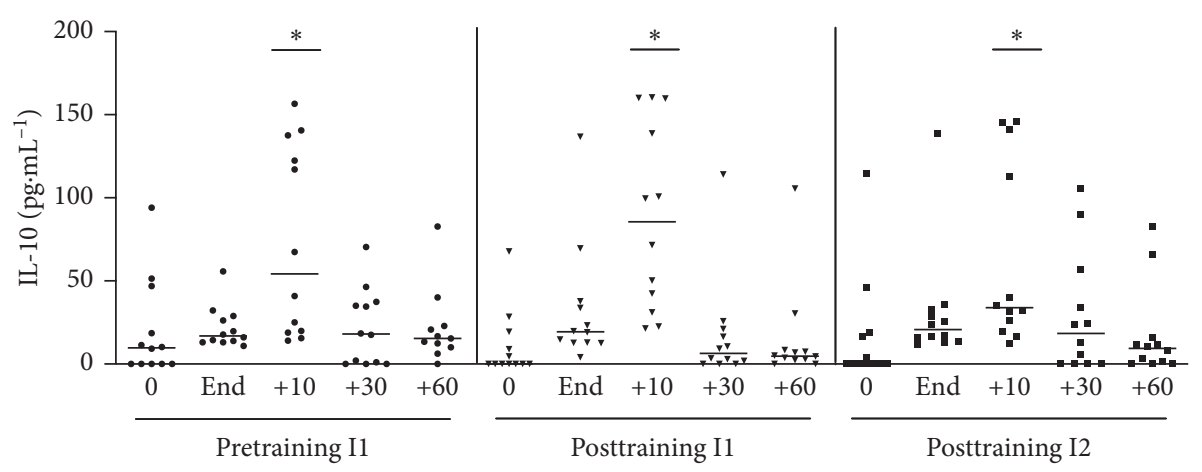

Figure 5: IL-10 plasmatic concentration at pretraining and posttraining, before, after, 10, 30, and 60 min after acute exercise. Pretraining (physical exercise performed until fatigue at anaerobic threshold (I1) before 6 weeks of training); posttraining 1 (aerobic acute exercise performed at the same absolute intensity (I1) and duration of pretraining) and posttraining 2 (after aerobic acute exercise performed until fatigue at anaerobic threshold of posttraining (I2)). ${ }^{*} p<0.05$ from resting period (0) for the same situation. $n=12$.

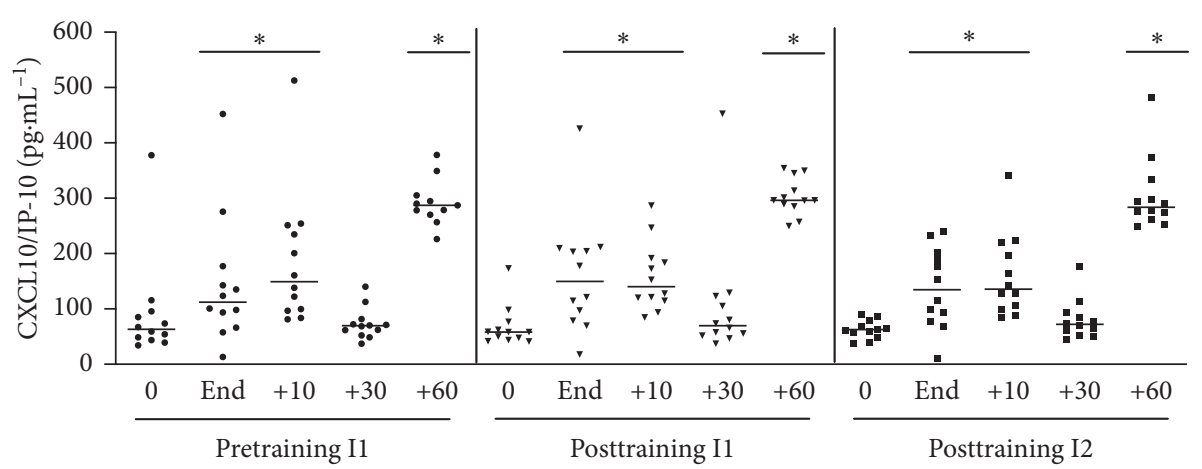

FIGURE 6: Plasma level of CXCL10/IP-10. Pretraining (physical exercise performed until fatigue at anaerobic threshold (I1) before 6 weeks of training); posttraining 1 (aerobic acute exercise performed at the same absolute intensity (I1) and duration of pretraining) and posttraining 2 (after aerobic acute exercise performed until fatigue at anaerobic threshold of posttraining (I2)). ${ }^{*} p<0.05$ from resting period (0) for the same situation. $n=12$.

a significant increase in circulating concentration of the cytokines IL-6, TNF- $\alpha$, sTNFR1, IL-10, CXCL10, BDNF, leptin, resistin, and adiponectin in plasma, albeit with different kinetics of increase. (iii) For the first time, we were able to show that six weeks of moderate aerobic exercise training at AT was able to modulate acute exercise-induced 


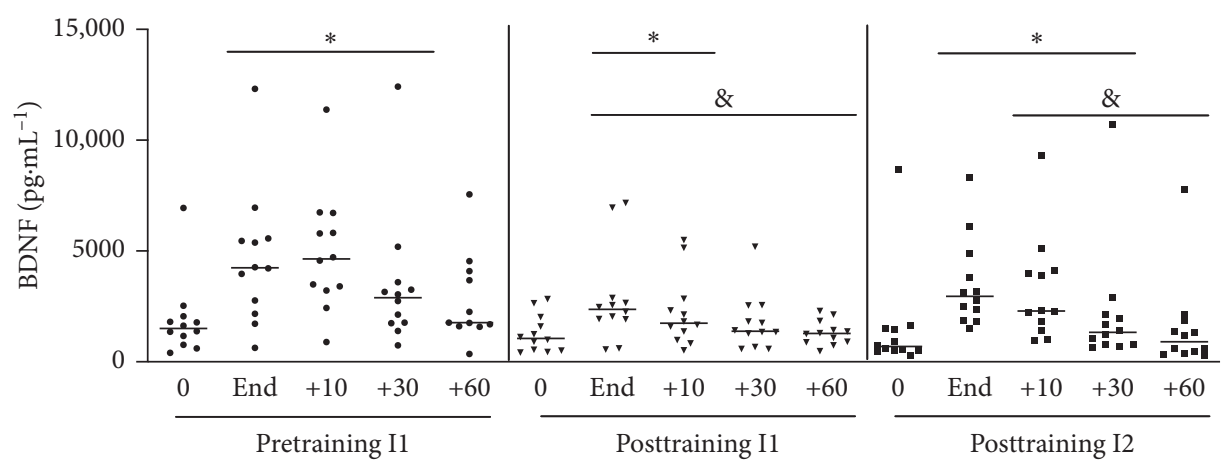

Figure 7: Plasma levels of BDNF. Pretraining (physical exercise performed until fatigue at anaerobic threshold (I1) before 6 weeks of training); posttraining 1 (aerobic acute exercise performed at the same absolute intensity (I1) and duration of pretraining) and posttraining 2 (after aerobic acute exercise performed until fatigue at anaerobic threshold of posttraining (I2)). ${ }^{*} p<0.05$ from resting period (0) for the same situation; ${ }^{\&} p<0.05$ from pretraining for the same time point; $n=12$.

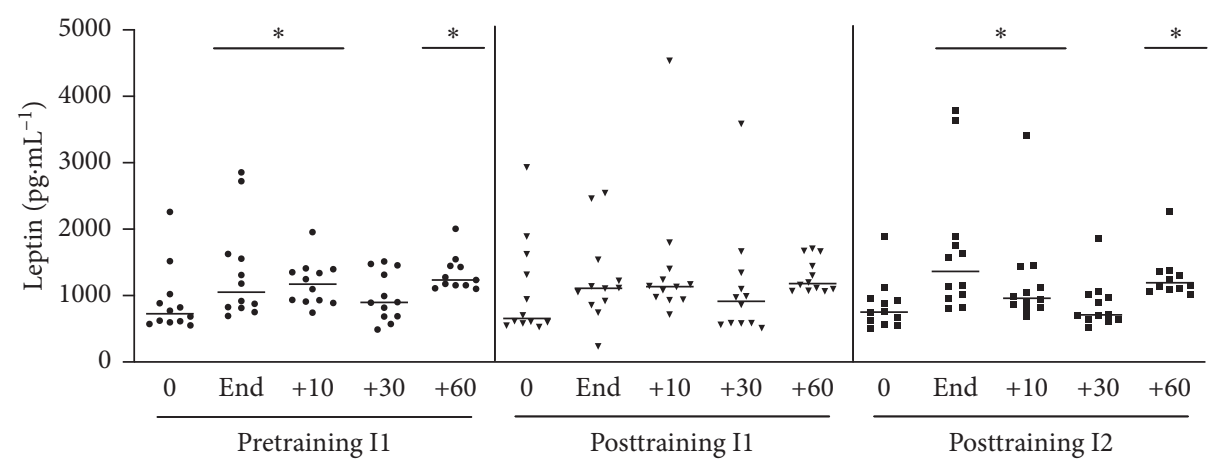

Figure 8: Leptin levels after acute physical exercise: the leptin levels were measured before and after exercise at times of 10, 30, and 60 minutes in postexercise recovery. Pretraining (physical exercise performed until fatigue at anaerobic threshold (I1) before 6 weeks of training); posttraining 1 (aerobic acute exercise performed at the same absolute intensity (I1) and duration of pretraining) and posttraining 2 (after aerobic acute exercise performed until fatigue at anaerobic threshold of posttraining (I2)). ${ }^{*} p<0.05$ from resting period (0) for the same situation; $n=12$.

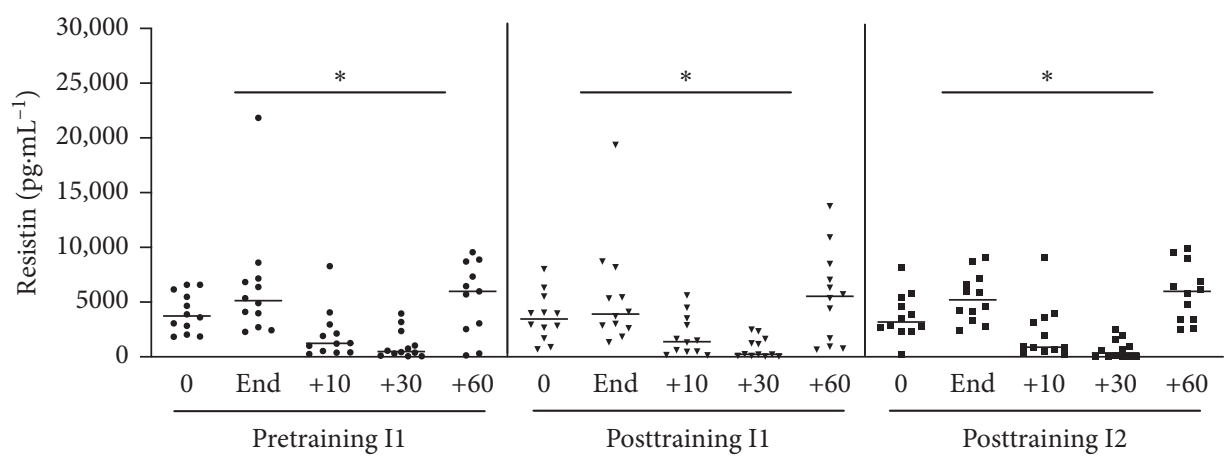

Figure 9: Resistin level after acute physical exercise: the resistin levels were measured before and after exercise at times of 10, 30, and 60 minutes in postexercise recovery. Pretraining (physical exercise performed until fatigue at anaerobic threshold (I1) before 6 weeks of training); posttraining 1 (aerobic acute exercise performed at the same absolute intensity (I1) and duration of pretraining) and posttraining 2 (after aerobic acute exercise performed until fatigue at anaerobic threshold of posttraining (I2)). ${ }^{*} p<0.05$ from resting period (0) for the same situation; $n=12$. 


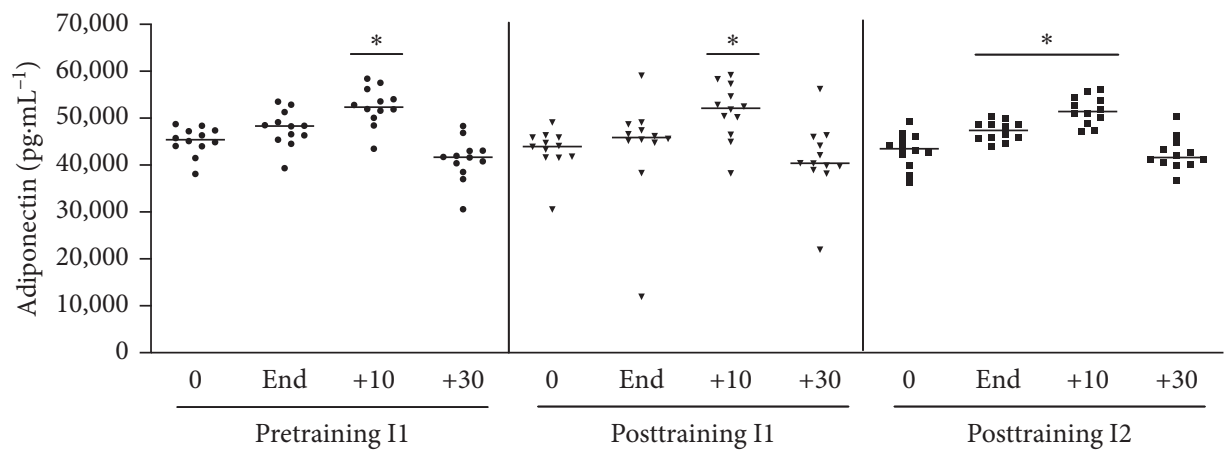

Figure 10: Plasma adiponectin levels after acute physical exercise: the plasma adiponectin levels were measured before and after exercise at times of 10,30, and 60 minutes in postexercise recovery. Pretraining (physical exercise performed until fatigue at anaerobic threshold (I1) before 6 weeks of training); posttraining 1 (aerobic acute exercise performed at the same absolute intensity (I1) and duration of pretraining) and posttraining 2 (after aerobic acute exercise performed until fatigue at anaerobic threshold of posttraining (I2)). ${ }^{*} p<0.05$ from resting period ( 0 ) for the same situation; $n=12$.

elevation of certain cytokines in such way that the elevation of plasma levels of certain cytokines (IL-6, sTNFR1, BDNF, and leptin) was altered by aerobic training, but not others (TNF- $\alpha$, IL-10, CXCL10, resistin, and adiponectin).

In this study, six weeks of aerobic training performed at $75 \%$ of $\mathrm{VO}_{2 \text { PEAK }}$ induced a significative improvement of $11.2 \pm 6.6 \%$ and a medium ES $(\mathrm{ES}=1.06)$ of exercise capacity, as assessed by maximal oxygen consumption. All participants showed an improvement of maximal oxygen consumption and power at AT $(14.7 \pm 8.9 \%$ improvement of initial AT) indicating the effects of aerobic training in AT and maximal oxygen consumption. This was a significant gain of exercise capacity in a short period of time and is comparable to other studies evaluating increase in performance [60]. Therefore, the findings of the current study may be extrapolated to other types of training with similar gain of performance.

In our study, acute exercise to fatigue was associated with an increase in circulating concentrations of several cytokines, including IL-6, TNF- $\alpha$, sTNFR1, IL-10, CXCI10/IP$10, \mathrm{BDNF}$, leptin, adiponectin, and resistin. This elevation is similar to that found by other studies [28-30, 61-63] and is consistent with the idea that acute exercise may function as an acute inflammatory stimulus [64-66]. Elevated cytokines included proinflammatory molecules (IL-6, TNF- $\alpha$ ), molecules with anti-inflammatory activity (IL-10, sTNFR1), adipokines (leptin, adiponectin, resistin), and neurotrophic factors (BDNF). A meta-analysis study showed that resting concentrations of peripheral blood BDNF were higher after training process. Subgroup analyses also suggested a significant effect in aerobic but not resistance training interventions. It appears that aerobic but not resistance training interventions increased resting BDNF concentrations in peripheral blood chronically [67].

In our study, the exercise session was able to elevate the levels of leptin. Physical exercise and/or training can reduce fat mass, play a significant role in energy expenditure, and affect hormonal concentrations (insulin, cortisol, growth hormone, catecholamines, testosterone, etc.) and metabolites (free fatty acids, lactic acid, triglycerides, etc.). For all these reasons we believe that physical exercise and training could modify the leptin response depending on several factors such as intensity and duration of exercise session [68].

Recently, an elegant paper using "omics" analysis investigated $2,15,30$, and 60 minutes after the end of aerobic exercise and showed an acute elevation in some molecules [69].

As example of the cytokine responses to exercise, the concentration of IL- 6 peaked at the end of the acute exercise protocol. Thereafter, the concentration of this cytokine was kept high until 30 minutes, with a drop at 60 minutes of the recovery period, although it was still higher than basal levels (see Figure 2). An increase of IL-6 concentration in plasma after exercise has been shown using different exercise protocols [70-72]. The increase of IL-6 in plasma appears to depend on exercise intensity and duration [32] and is consistent with the suggested role of this cytokine in mobilizing energy and muscle hypertrophy [63]. The role of other molecules in the context of exercise is less understood, but it is believed that this acute inflammatory response may be relevant for metabolic and adaptive responses of the muscle, liver, and adipose tissue [73].

There were some differences in the time point at which the elevation of the concentration of cytokines occurred, when compared with other authors [27, 31, 32]. These differences may be explained by the type and intensity of exercise, which will determine the intensity of the inflammatory stimulation. In this regard, it is clear that our acute exercise protocol was capable of inducing a robust inflammatory stimulus followed by systemic increase in concentration of certain cytokines.

In the posttraining protocol, cytokine responses induced by acute exercise followed two general patterns: (i) There were cytokines which were elevated in a similar manner before and after training, if the individuals were subjected to the same relative or absolute acute exercise, and these included TNF- $\alpha$, IL-10, CXCL10, resistin, and adiponectin. (ii) There were cytokines which responded in a different manner, if the posttraining were performed at the same absolute level of intensity of acute exercise as that given before the training; these included IL-6, sTNFR1, and leptin. 
In these individuals, the elevation of concentration of cytokines was smaller, or the return to baseline was faster. In contrast, they still had a strong response that was similar to that of the pretraining period, if they were again exposed to the same relative intensity level; i.e., there was training for cytokine responses as there was training for exercise.

Evaluation of these data clearly show that physical training induced no absolute and straightforward changes in cytokine responses to acute inflammatory stimulation (acute exercise); i.e., there were no major changes in proinflammatory vs anti-inflammatory molecules, or molecules that most likely induce metabolic changes versus those that do not. Indeed, the molecules that undergo training in their response are rather diverse in their nature and molecular actions. IL- 6 is clearly active on the muscle and metabolic tissues [62], whereas sTNFR1 would tend to counteract these effects by decreasing TNF- $\alpha$ function by blocking its ability to activate its receptors on cells in vivo. Leptin has strong metabolic effects, but so do resistin and adiponectin [74]. Therefore, although we do not completely understand the meaning of the elevation of any particular cytokine, the results do show that the stress induced by exercise over the immune system can be modulated by physical training. Another important aspect from these findings and that is hard to be discussed is that, in a fatigue point at the AT intensity, the inflammatory response appears to be the same. This finding suggests that, independent of the physical fitness level of a given individual, the inflammatory response to maximal effort is the same, revealing an interesting setpoint of regulation of immune responses.

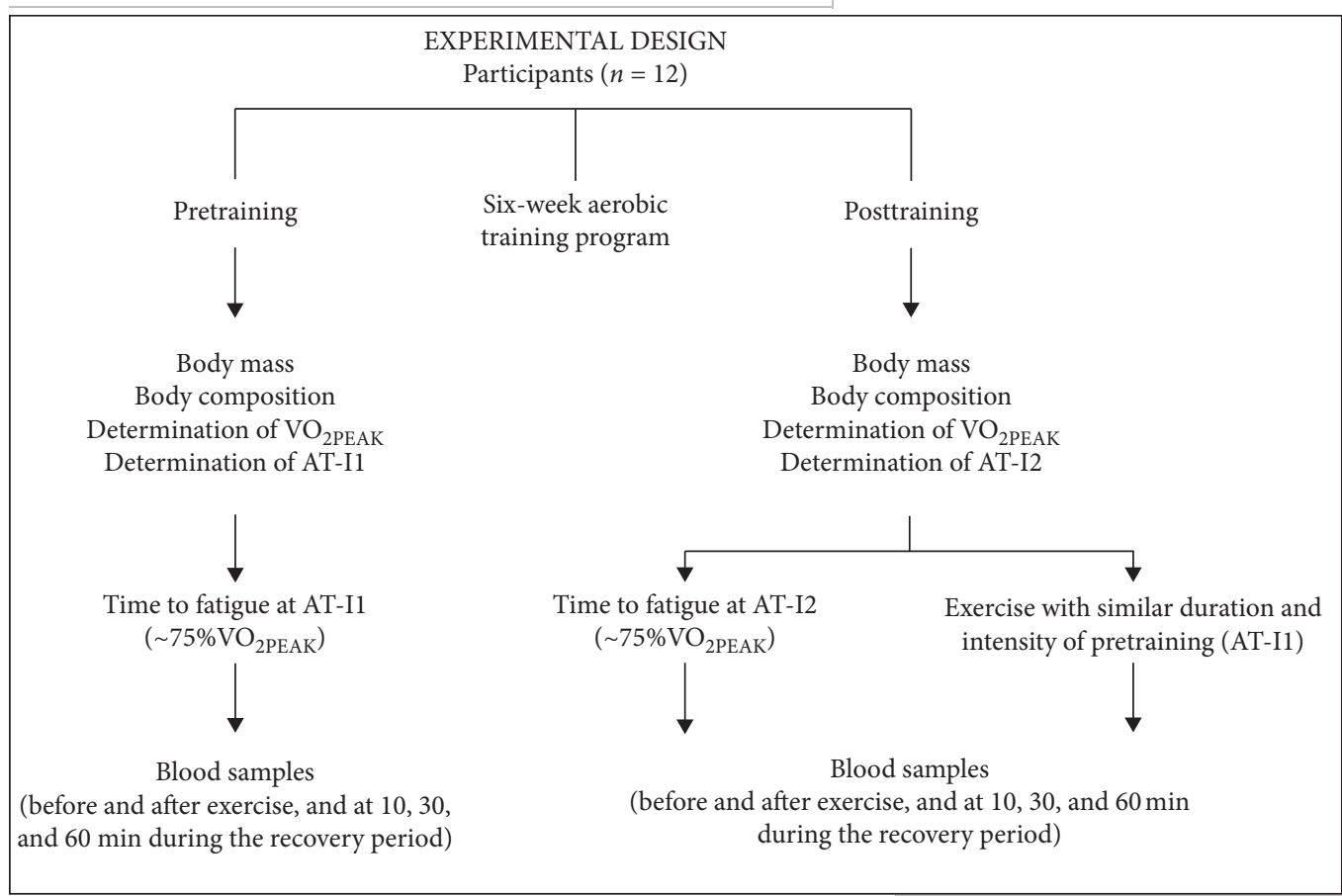

At this stage, it is not simple to correlate the relevance of the training of cytokine responses to any beneficial effects of exercise on health or disease. It is possible that training of cytokine responses may favor the ability of humans to deal better with chronic inflammatory and metabolic diseases, because it tunes down-but does not completely inhibit-the intensity of inflammation. A similar beneficial effect may occur in the context of infection. Indeed, inflammatory responses are necessary for infection but may cause tissue damage if excessive [70]. By training the immune system and fine-tuning cytokines, chronic regular exercise may modulate the function of the immune system in a beneficial manner. This is an interesting concept because it suggests that the immune system performs a much more physiological function (still to be defined) than before. Indeed, the concept of the immune system was developed from the concept of fighting infection; i.e., the immune system has been defined much more by its pathophysiological than physiological functions [73, 75]. The fact that there are significant changes of immunological responses to exercise suggests that the immune system may play a much more important physiological role than previously understood [75]. This possibility is compounded by findings that immunological responses are modulated by diet and by the microbiota in a normal physiological manner; i.e., the function of the immune system is modulated under normal physiology without the need for disease [75]. Much more knowledge is required to define the exact physiology of the immune system and how it interacts with other physiological systems.

A major limitation of the current study, and indeed of any study that evaluates particular exercise or training protocols, is that the findings may be specific to the training protocol, acute exercise, or age of participants under investigation. We studied inflammatory responses to exercise at the fatigue point at AT intensity, in an attempt to normalize exercise load before and after training. However, our 
studies do show a physiological effect of exercise on the immune system and suggest that understand the physiology of the immune system may be useful to understand its relevance to disease.

There are many speculations and expectations regarding the benefits of chronic physical exercise to health and particularly to the immune system. In conclusion, our results show that moderate chronic aerobic exercise causes significant "training" for certain cytokine responses, including IL-6, sTNFR1, BDNF, and leptin. Trained immunity is known to be relevant in the context of infection but may contribute to the pathogenesis of certain conditions, such as sepsis [44]. Whether the ability of exercise to train immune responses is relevant in the context of health and disease is not yet known but clearly deserves further investigation.

\section{Data Availability}

Data cannot be shared regarding this paper.

\section{Conflicts of Interest}

The authors declare that they have no conflicts of interest regarding this article.

\section{Acknowledgments}

This work was supported by grants from Coordenação de Aperfeiçoamento de Pessoal do Ensino Superior (CAPES), Conselho Nacional de Desenvolvimento Científico e Tecnológico $(\mathrm{CNPq})$, and Fundação de Amparo à Pesquisa do Estado de Minas Gerais (FAPEMIG).

\section{References}

[1] F. W. Booth, S. E. Gordon, C. J. Carlson, and M. T. Hamilton, "Waging war on modern chronic diseases: primary prevention through exercise biology," Journal of Applied Physiology, vol. 88, no. 2, pp. 774-787, 2000.

[2] F. W. Booth, C. K. Roberts, and M. J. Laye, "Lack of exercise is a major cause of chronic diseases," Comprehensive Physiology, vol. 2, no. 2, pp. 1143-1211, 2012.

[3] J. E. Clark, "An overview of the contribution of fatness and fitness factors, and the role of exercise, in the formation of health status for individuals who are overweight," Journal of Diabetes \& Metabolic Disorders, vol. 11, p. 19, 2012.

[4] D. J. Green, G. O'Driscoll, M. J. Joyner, and N. T. Cable, "Exercise and cardiovascular risk reduction: time to update the rationale for exercise?" Journal of Applied Physiology, vol. 105, no. 2, pp. 766-768, 2008.

[5] K. Karstoft and B. K. Pedersen, "Exercise and type 2 diabetes: focus on metabolism and inflammation," Immunology and Cell Biology, vol. 94, no. 2, pp. 146-150, 2015.

[6] M. Riddell and B. A. Perkins, "Exercise and glucose metabolism in persons with diabetes mellitus: perspectives on the role for continuous glucose monitoring," Journal of Diabetes Science and Technology, vol. 3, no. 4, pp. 914-923, 2009.

[7] D. M. Swift, N. M. Johannsen, C. J. Lavie, C. P. Earnest, and T. S. Church, "The role of exercise and physical activity in weight loss and maintenance," Progress in Cardiovascular Disease, vol. 56, no. 4, pp. 441-447, 2014.
[8] F. Mattsuch, B. Dufaux, O. Heine, I. Mertens, and R. Rost, "Reduction of the plasma concentration of C-reactive protein following nine months of endurance training," International Journal of Sports Medicine, vol. 21, no. 1, pp. 21-24, 2000.

[9] S. Adamopoulos, J. Parissis, C. Kroupis et al., "Physical training reduces peripheral markers of inflammation in patients with chronic heart failure," European Heart Journal, vol. 22, no. 9, pp. 791-797, 2001.

[10] S. Gielen, V. Adams, S. Mobius-Winkler et al., "Anti-inflammatory effects of exercise training in the skeletal muscle of patients with chronic heart failure," Journal of the American College of Cardiology, vol. 42, no. 5, pp. 861-868, 2003.

[11] A. M. W. Petersen and B. K. Pedersen, "The anti-inflammatory effect of exercise," Journal of Applied Physiology, vol. 98, no. 4, pp. 1154-1162, 2005.

[12] A. Abassi, M. Hauth, M. Walter et al., "Exhaustive exercise modifies different gene expression profiles and pathways in LPS-stimulated and un-stimulated whole blood cultures," Brain, Behavior, and Immunity, vol. 39, pp. 130-141, 2014.

[13] K. V. Der Borght, R. Havekes, T. Bos, B. J. L. Eggen, and E. A. V. Der Zee, "Exercise improves memory acquisition and retrieval in the Y-maze task: relationship with hippocampal neurogenesis," Behavioral Neuroscience, vol. 121, no. 2, pp. 324-334, 2007.

[14] E. E. Bernstein and R. J. McNally, "Acute aerobic exercise helps overcome emotion regulation deficits," Cognition and Emotion, vol. 31, no. 4, pp. 834-843, 2016.

[15] J. Stessman, R. Hammerman-Rozenberg, A. Cohen, E. EinMor, and J. M. Jacobs, "Physical activity, function, and longevity among the very old," Archives of Internal Medicine, vol. 169, no. 16, pp. 1476-1483, 2009.

[16] K. Krueger, A. Fromme, C. Korsukewitz et al., "High impact running improves learning," Neurobiology of Learning and Memory, vol. 87, no. 4, pp. 597-609, 2007.

[17] X. Lin, X. Zhang, J. Guo et al., "Effects of exercise training on cardiorespiratory fitness and biomarkers of cardiometabolic health: a systematic review and meta-analysis of randomized controlled trials," Journal of the American Heart Association, vol. 4, no. 7, Article ID e002014, 2015.

[18] D. C. Nieman, "Exercise, infection, and immunity," International Journal of Sports Medicine, vol. 15, no. 3, pp. S131S141, 1994.

[19] D. C. Nieman, "Exercise, upper respiratory tract infection, and the immune system," Medicine \& Science in Sports \& Exercise, vol. 26, no. 2, pp. 128-139, 1994.

[20] D. C. Nieman, D. A. Henson, L. L. Smith et al., "Cytokine changes after a marathon race," Journal of Applied Physiology, vol. 91, no. 1, pp. 109-114, 2001.

[21] B. K. Pedersen and L. Hoffman-Goetz, "Exercise and the immune system: regulation, integration, and adaptation," Physiological Reviews, vol. 80, no. 3, pp. 1055-1081, 2000.

[22] K. Suzuki, M. Yamada, S. Kurakake et al., "Circulating cytokines and hormones with immunosuppressive but neutrophil-priming potentials rise after endurance exercise in humans," European Journal of Applied Physiology, vol. 81, no. 4, pp. 281-287, 2000.

[23] A. D. Toft, L. B. Jensen, H. Bruunsgaard et al., "Cytokine response to eccentric exercise in young and elderly humans," American Journal of Physiology-Cell Physiology, vol. 283, no. 1, pp. C289-C295, 2002.

[24] D. C. Nieman, J. M. Davis, D. A. Henson et al., "Carbohydrate ingestion influences skeletal muscle cytokine mRNA and plasma cytokine levels after a 3-h run," Journal of Applied Physiology, vol. 94, no. 5, pp. 1917-1925, 2003. 
[25] K. Suzuki, S. Nakaji, M. Yamada et al., "Impact of a competitive marathon race on systemic cytokine and neutrophil responses," Medicine \& Science in Sports \& Exercise, vol. 35, no. 2, pp. 348-355, 2003.

[26] J. M. Peake, K. Suzuki, G. Wilson et al., "Exercise-induced muscle damage, plasma cytokines, and markers of neutrophil activation," Medicine \& Science in Sports \& Exercise, vol. 37, no. 5, pp. 737-745, 2005.

[27] M. W. Kakanis, J. M. Peake, E. W. Brenu et al., "The open window of susceptibility to infection after acute exercise in healthy young male elite athletes," Exercise Immunology Review, vol. 16, pp. 119-137, 2010.

[28] J. P. Drenth, R. J. Krebbers, J. Bijzet, and J. W. Van der Meer, "Increased circulating cytokine receptors and ex vivo interleukin-1 receptor antagonist and interleukin-1 beta production but decreased tumour necrosis factor-alpha production after a 5-km run," European Journal of Clinical Investigation, vol. 28, no. 10, pp. 866-872, 1998.

[29] J. Widenfalk, L. Olson, and P. Thoren, "Deprived of habitual running, rats downregulate BDNF and TrkB messages in the brain," Journal of Neuroscience Research, vol. 34, no. 3, pp. 125-132, 1999.

[30] E. Goldhammer, A. Tanchilevitch, I. Maor, Y. Beniamini, U. Rosenschein, and M. Sagiv, "Exercise training modulates cytokines activity in coronary heart disease patients," International Journal of Cardiology, vol. 100, no. 1, pp. 93-99, 2005.

[31] J. M. Peake, K. Suzuki, M. Hordern, G. Wilson, K. Nosaka, and J. S. Coombes, "Plasma cytokine changes in relation to exercise intensity and muscle damage," European Journal of Applied Physiology, vol. 95, no. 5-6, pp. 514-521, 2005.

[32] J. M. Peake, P. D. Gatta, K. Suzuki, and D. C. Nieman, "Cytokine expression and secretion by skeletal muscle cells: regulatory mechanisms and exercise effects," Exercise Immunology Review, vol. 21, pp. 8-25, 2015.

[33] J. M. Hinkley, A. R. Konopka, M. K. Suer, and M. P. Harber, "Short-term intense exercise training reduces stress markers and alters the transcriptional response to exercise in skeletal muscle," American Journal of Physiology-Regulatory, Integrative and Comparative Physiology, vol. 312, no. 2, pp. R426-R433, 2017.

[34] N. Hiscock, M. H. Chan, T. Bisucci, I. A. Darby, and M. A. Febbraio, "Skeletal myocytes are a source of interleukin6 mRNA expression and protein release during contraction: evidence of fiber type specificity," FASEB Journal, vol. 18, no. 9, pp. 992-994, 2004.

[35] C. P. Fischer, "Interleukin-6 in acute exercise and training: what is the biological relevance?" Exercise Immunology Review, vol. 12, pp. 6-33, 2006.

[36] S. K. Gill, A. Teixeira, L. Rama et al., "Circulatory endotoxin concentration and cytokine profile in response to exertionalheat stress during a multi-stage ultra-marathon competition," Exercise Immunology Review, vol. 21, pp. 114-128, 2015.

[37] S. P. Hoekstra, N. C. Bishop, and C. A. Leicht, "Can intervals enhance the inflammatory response and enjoyment in upperbody exercise?" European Journal of Applied Physiology, vol. 117, no. 6, pp. 1155-1163, 2017.

[38] A. Margeli, K. Skenderi, M. Tsironi et al., "Dramatic elevations of interleukin-6 and acute-phase reactants in athletes participating in the ultradistance foot race spartathlon: severe systemic inflammation and lipid and lipoprotein changes in protracted exercise," Journal of Clinical Endocrinology and Metabolism, vol. 90, no. 7, pp. 3914-3918, 2005.

[39] E. R. Ropelle, M. B. Flores, D. E. Cintra et al., "IL-6 and IL 10 anti-inflammatory activity links exercise to hypothalamic insulin and leptin sensitivity through IKKb and ER stress inhibition," PLoS Biology, vol. 8, no. 8, Article ID e1000465, 2010.

[40] H.-K. Kim, M. Konishi, M. Takahashi et al., "Effects of acute endurance exercise performed in the morning and evening on inflammatory cytokine and metabolic hormone responses," PLoS One, vol. 10, no. 9, Article ID e0137567, 2015.

[41] A. Strömberg, E. Rullman, E. Jansson, and T. Gustafsson, "Exercise-induced upregulation of endothelial adhesion molecules in human skeletal muscle and number of circulating cells with remodeling properties," Journal of Applied Physiology, vol. 122, no. 5, pp. 1145-1154, 2017.

[42] A. Nunes-Silva, P. T. Bernardes, B. M. Rezende et al., "Treadmill exercise induces neutrophil recruitment into muscle tissue in a reactive oxygen species-dependent manner. An intravital microscopy study," PLoS One, vol. 9, no. 5, Article ID e96464, 2014.

[43] D. R. Lacerda, M. M. Moraes, A. Nunes-Silva et al., "Aerobic training reduces immune cell recruitment and cytokine levels in adipose tissue in obese mice," Applied Physiology, Nutrition, and Metabolism, vol. 44, no. 5, pp. 512-520, 2018.

[44] M. G. Netea, L. A. B. Joosten, E. Latz et al., "Trained immunity: a program of innate immune memory in health and disease," Science, vol. 352, no. 6284, Article ID aaf1098, 2016.

[45] A. Philp, A. L. Macdonald, H. Carter et al., "Maximal lactate steady state as a training stimulus," International Journal of Sports Medicine, vol. 29, no. 6, pp. 475-479, 2008.

[46] H. Carter, A. M. Jones, and J. H. Doust, "Effect of 6 weeks of endurance training on the lactate minimum speed," Journal of Sports Sciences, vol. 17, no. 12, pp. 957-967, 1999.

[47] A. S. Jackson and M. L. Pollock, "Generalized equations for predicting body density of men," British Journal of Nutrition, vol. 40, no. 3, pp. 497-504, 1978.

[48] V. A. Convertino, L. E. Armstrong, E. F. Coyle et al., "American college of sports medicine position stand. Exercise and fluid replacement," Medicine \& Science in Sports \& Exercise, vol. 28, no. 1, pp. 1-7, 1996.

[49] B. Balke and R. W. Ware, "An experimental study of physical fitness of air force personnel," United States Armed Forces Medical Journal, vol. 10, no. 6, pp. 675-688, 1959.

[50] G. Borg, "Psychophysical bases of perceived exertion," Medicine \& Science in Sports \& Exercise, vol. 14, no. 5, pp. 377-381, 1982.

[51] D. Riebe, J. K. Ehrman, and G. Liguori, Guidelines for Exercise Testing and Prescription, American College of Sports Medicine (ACSM), Indianapolis, IN, USA, 2009.

[52] T. T. Mendes, G. P. Ramos, C. F. Wilke et al., "Six weeks of aerobic training improves VO2max and MLSS but does not improve the time to fatigue at the MLSS," European Journal of Applied Physiology, vol. 113, no. 4, pp. 965-973, 2012.

[53] H. Heck, A. Mader, G. Hess et al., "Justificationof the 4-mmol/ 1 lactate threshold," International Journal of Sports Medicine, vol. 6, no. 3, pp. 117-130, 1985.

[54] R. Beneke, "Methodological aspects of maximal lactate steady state-implications for performance testing," European Journal of Applied Physiology, vol. 89, no. 1, pp. 95-99, 2003.

[55] O. Faude, W. Kindermann, and T. Meyer, "Lactate threshold concepts: how valid are they?" Sports Medicine, vol. 39, no. 6, pp. 469-490, 2009.

[56] B. Baron, T. D. Noakes, J. Dekerle et al., "Why does exercise terminate at the maximal lactate steady state intensity?" British Journal of Sports Medicine, vol. 42, no. 10, pp. 528-533, 2008. 
[57] D. B. Dill and D. L. Costill, "Calculation of percentage changes in volumes of blood, plasma, and red cells in dehydration," Journal of Applied Physiology, vol. 37, no. 2, pp. 247-248, 1974.

[58] J. Cohen, Statistical Power Analysis for the Behavioral Sciences, Lawrence Earlbaum Associates, Hillsdale, NJ, USA, 2nd edition, 1988.

[59] W. G. Hopkins, S. W. Marshall, A. M. Batterham, and J. Hanin, "Progressive statistics for studies in sports medicine and exercise science," Medicine \& Science in Sports \& Exercise, vol. 41, no. 1, pp. 3-13, 2009.

[60] M. Sloth, D. Sloth, K. Overgaard, and U. Dalgas, "Effects of sprint interval training on VO2max and aerobic exercise performance: a systematic review and meta-analysis," Scandinavian Journal of Medicine \& Science in Sports, vol. 23, no. 6, pp. e341-e352, 2013.

[61] B. K. Pedersen and A. D. Toft, "Effects of exercise on lymphocytes and cytokines," British Journal of Sports Medicine, vol. 34, no. 4, pp. 246-251, 2000.

[62] B. K. Pedersen and M. A. Febbraio, "Muscle as an endocrine organ: focus on muscle-derived interleukin-6," Physiological Reviews, vol. 88, no. 4, pp. 1379-1406, 2008.

[63] B. K. Pedersen and M. A. Febbraio, "Muscles, exercise and obesity: skeletal muscle as a secretory organ," Nature Reviews Endocrinology, vol. 8, no. 8, pp. 457-465, 2012.

[64] A. Fortunato, W. Pontes, D. Souza et al., "Strength training session induces important changes on physiological, immunological, and inflammatory biomarkers," Journal of Immunology Research, vol. 2018, Article ID 9675216, 12 pages, 2018.

[65] L. S. Marcucci-Barbosa, F. d. A. D. Martins-Junior, L. F. Lobo et al., "The effects of strength training session with different types of muscle action on white blood cells counting and Th1/ Th2 response," Sport Sciences for Health, vol. 16, pp. 239-248, 2019.

[66] L. S. Marcucci-Barbosa, F. Martins-Junior, L. F. Lobo et al., " $10 \mathrm{~km}$ running race induces an elevation in the plasma myokine level of nonprofessional runners," Sport Sciences for Health, vol. 16, pp. 313-321, 2019.

[67] A. Dinoff, N. Herrmann, W. Swardfager et al., "The effect of exercise training on resting concentrations of peripheral brain-derived neurotrophic factor (BDNF): 2016. A metaanalysis," PLoS One, vol. 11, no. 9, pp. 1-21, 2016.

[68] A. Bouassida, D. Zalleg, S. Bouassida et al., "Leptin, its implication in physical exercise and training: a short review," Journal of Sports Science and Medicine, vol. 5, no. 2, pp. 172-181, 2006.

[69] K. Contrepois, S. Wu, K. J. Moneghetti et al., "Molecular choreography of acute exercise," Cell, vol. 181, no. 5, pp. 1112-1130, 2020.

[70] F. B. Benatti and B. K. Pedersen, "Exercise as an anti-inflammatory therapy for rheumatic diseases-myokine regulation," Nature Reviews Rheumatology, vol. 11, no. 2, pp. 86-97, 2014.

[71] B. K. Pedersen and B. Saltin, "Exercise as medicine-evidence for prescribing exercise as therapy in 26 different chronic diseases," Scandinavian Journal of Medicine \& Science in Sports, vol. 25, no. 3, pp. 1-72, 2015.

[72] H. G. Nielsen, O. Øktedalen, P. Opstad, and T. Lyberg, "Plasma cytokine profiles in long-term strenuous exercise," Journal of Sports Medicine, vol. 2016, Article ID 7186137, 7 pages, 2016.

[73] M. A. Sugimoto, L. P. Sousa, V. Pinho, M. Perretti, and M. M. Teixeira, "Resolution of inflammation: what controls its onset?” Frontiers in Immunology, vol. 7, p. 160, 2016.

[74] M. I. Jonas, A. Kurylowicz, Z. Bartoszewicz et al., "Adiponectin/resistin interplay in serum and in adipose tissue of obese and normal-weight individuals," Diabetology \& Metabolic Syndrome, vol. 9, p. 95, 2017.

[75] R. F. Marques, P. E. Marques, R. Guabiraba, and M. M. Teixeira, "Exploring the homeostatic and sensory roles of the immune system," Frontiers in Immunology, vol. 7, pp. 1-7, 2016. 